\title{
Urinary Free Metanephrines for Diagnosis of Pheochromocytoma and Paraganglioma
}

\author{
Jiyeon Ahn ${ }^{1,2}$, Ji Yun Park ${ }^{1}$, Gyuri Kim ${ }^{1}$, Sang-Man Jin ${ }^{1}$, Kyu Yeon Hur ${ }^{1}$, Soo-Youn Lee ${ }^{3}$, Jae Hyeon Kim ${ }^{1}$ \\ ${ }^{1}$ Division of Endocrinology and Metabolism, Department of Medicine, Samsung Medical Center, Sungkyunkwan University \\ School of Medicine, Seoul; ${ }^{2}$ Division of Endocrinology and Metabolism, Department of Internal Medicine, Myongji Hospital, \\ Hanyang University College of Medicine, Goyang; ${ }^{3}$ Department of Laboratory Medicine and Genetics, Samsung Medical \\ Center, Sungkyunkwan University School of Medicine, Seoul, Korea
}

Background: Pheochromocytoma and paraganglioma (PPGL) is diagnosed through biochemical confirmation of excessive catecholamines in urine and plasma. Recent technological developments have allowed us to measure urinary free metanephrines; however, the diagnostic accuracy of these new methods and the diagnostic cutoff values have not been evaluated.

Methods: This is a retrospective study of 595 subjects, including 71 PPGL cases and 524 controls. PPGL was based on pathological confirmation. Subjects with no evidence of PPGL over 2 years were included in the control group.

Results: Urinary free metanephrines yielded similar area under the curve (AUC) to urinary fractionated metanephrines and plasma free metanephrines. However, urinary free normetanephrine yielded a better AUC than did urinary fractionated normetanephrine. The optimal cutoff for urinary free metanephrine and normetanephrine corrected for urinary creatinine yielded $97.2 \%$ sensitivity and $98.1 \%$ specificity.

Conclusion: Urinary free metanephrines are a reliable method for diagnosing PPGL in Asian populations compared with existing biochemical methods.

Keywords: Metanephrine; Normetanephrine; Pheochromocytoma; Paraganglioma

\section{INTRODUCTION}

Pheochromocytoma and paraganglioma (PPGL) is a rare tumor that originates in chromaffin cells, which produce catecholamine [1]. PPGL can cause various symptoms, but it is difficult to diagnose because an asymptomatic phenotype also is common [2,3]. Additionally, as the number of imaging tests increases, detection of adrenal incidentaloma increases [4]. Undiagnosed PPGL can be fatal [5], so accurate biochemical testing is needed.

PPGL can be diagnosed by measuring the concentrations of catecholamines and their metabolites of metanephrines and vanillylmandelic acid [2]. Metanephrines in urine are mostly in sulfur-bonded (conjugated) form. However, PPGL has a high proportion of free (unconjugated) forms, especially normetanephrine [6]. Until recently, most centers measured fractionated total (free and sulfur-conjugated form) metanephrines. However, technological developments allow measurement of free
Received: 27 November 2020, Revised: 12 March 2021, Accepted: 12 April 2021

Corresponding author: Jae Hyeon Kim

Division of Endocrinology and Metabolism, Department of Medicine, Samsung

Medical Center, Sungkyunkwan University School of Medicine, 81 Irwon-ro, Gangnam-gu, Seoul 06351, Korea

Tel: $+82-2-3410-1580$, Fax: $+82-2-3410-0393$,

E-mail: jaehyeon@skku.edu

\section{Copyright $\odot 2021$ Korean Endocrine Society}

This is an Open Access article distributed under the terms of the Creative Commons Attribution Non-Commercial License (https://creativecommons.org/ licenses/by-nc/4.0/) which permits unrestricted non-commercial use, distribution, and reproduction in any medium, provided the original work is properly cited. 
forms alone [6-8]. A recent study on the efficacy of urinary free metanephrines in a Western population showed high sensitivity [9]. However, the diagnostic accuracy and optimal cutoff value for Asian populations have not been evaluated. In this study, we identified the accuracy and cutoff value of urinary free metanephrines in a Korean sample.

\section{METHODS}

We retrospectively reviewed data from patients who were tested for PPGL from August 31, 2013 through March 19, 2019 at Samsung Medical Center (Supplemental Fig. S1). The subjects were those with symptoms of suspected PPGL (e.g., headache, sweating, palpitation, sustained, or paroxysmal hypertension $[n=39])$ or with an adrenal mass on imaging $(n=773)$. The study protocol was approved by the Institutional Review Board (IRB) of Samsung Medical Center, and the need for informed consent was waived (IRB FILE No. 2020-03-094) because the study was retrospective in design and analyzed de-identified data.

The PPGL group was confirmed by pathological findings from surgically removed tumors. The control group consisted of subjects whose pathologies were confirmed to be non-PPGL $(n=71)$ and who were revealed to have normal adrenal glands upon biochemical and imaging tests over more than 2 years of follow-up $(n=453)$.

Urinary fractionated metanephrines, plasma free and urinary free metanephrines were measured by liquid chromatography tandem mass spectrometry (LC-MS/MS). The protocols for measuring metanephrines are the same as previously reported $[10,11]$. Additionally, when the 24-hour urinary creatinine excretion amount was 1.4 to $2.35 \mathrm{~g}$ /day in males or 0.74 to $1.57 \mathrm{~g} /$ day in females, the sample was classified as optimally collected according to the reference value of the test reagent.

Receiver operating characteristic (ROC) curves were used to assess cutoff values that yielded the highest sensitivity and specificity. Differences in sensitivities and specificities were compared using McNemar's test. To calculate cutoff values for urinary metanephrines, the combination of urinary metanephrine and normetanephrine that yielded maximal accuracy was estimated using Youden's index method. All statistical tests were two-sided, and analyses were executed using SPSS version 18.0 (SPSS Inc., Chicago, IL, USA) and STATA version 15 (StataCorp., College Station, TX, USA). A $P$ value $<0.05$ was considered statistically significant.

Table 1. Baseline Characteristics of Patients and Controls

\begin{tabular}{|c|c|c|c|}
\hline Characteristic & Control $(n=524)$ & $\operatorname{PPGL}\left(n=71^{\mathrm{a}}\right)$ & $P$ value \\
\hline Male sex & $286(54.58)$ & $42(59.15)$ & 0.467 \\
\hline Age, yr & $55.00 \pm 10.59$ & $53.49 \pm 15.13$ & 0.287 \\
\hline BMI, $\mathrm{kg} / \mathrm{m}^{2}$ & $25.53 \pm 3.27$ & $23.49 \pm 3.29$ & $<0.001$ \\
\hline SBP, mm Hg & $129.38 \pm 30.35$ & $132.35 \pm 18.79$ & 0.422 \\
\hline DBP, mm Hg & $80.52 \pm 11.74$ & $82.39 \pm 15.38$ & 0.227 \\
\hline HTN & $237(45.23)$ & $64(90.14)$ & $<0.001$ \\
\hline DM & $144(27.48)$ & $13(18.31)$ & 0.100 \\
\hline Serum $\mathrm{Cr}, \mathrm{mg} / \mathrm{dL}$ & $0.84 \pm 0.20$ & $0.83 \pm 0.15$ & 0.728 \\
\hline BUN, mg/dL & $14.43 \pm 3.91$ & $14.05 \pm 4.82$ & 0.455 \\
\hline MDRD eGFR, $\mathrm{mL} / \mathrm{min} / 1.73 \mathrm{~m}^{2}$ & $93.04 \pm 19.70$ & $95.41 \pm 20.68$ & 0.345 \\
\hline Urinary free $\mathrm{MN}, \mu \mathrm{g} /$ day & $16.53 \pm 7.91$ & $332.08 \pm 801.36$ & $<0.001$ \\
\hline Urinary free $\mathrm{NMN}, \mu \mathrm{g} /$ day & $34.63 \pm 143.89$ & $885.48 \pm 3,549.64$ & $<0.001$ \\
\hline Urinary free $\mathrm{MN} / \mathrm{Cr}$ ratio, $\mu \mathrm{g} / \mathrm{g}$ & $14.13 \pm 7.87$ & $289.86 \pm 738.10$ & $<0.001$ \\
\hline Urinary free $\mathrm{NMN} / \mathrm{Cr}$ ratio, $\mu \mathrm{g} / \mathrm{g}$ & $29.18 \pm 96.41$ & $714.90 \pm 2,725.56$ & $<0.001$ \\
\hline
\end{tabular}

Values are expressed as number (\%) or mean \pm standard deviation.

PPGL, pheochromocytoma and paraganglioma; BMI, body mass index; SBP, systolic blood pressure; DBP, diastolic blood pressure; HTN, hypertension; DM, diabetes mellitus; Cr, creatinine; BUN, blood urea nitrogen; MDRD, Modification of Diet in Renal Disease; eGFR, estimated glomerular filtration rate; $\mathrm{MN}$, metanephrine; $\mathrm{NMN}$, normetanephrine.

apheochromocytoma $(n=68)$, paraganglioma $(n=3)$. 


\section{RESULTS}

After excluding patients who did not meet the study criteria, we included 595 subjects as either confirmed controls or PPGL cases (control $n=524$, PPGL $n=71$ ) (Supplemental Fig. S1). Subject baseline characteristics are shown in Table 1.

When metanephrine and normetanephrine were considered together, plasma and urinary metanephrines all showed high diagnostic performance (Table 2, Supplemental Tables S1, S2). Although urinary free metanephrine showed no significant difference in area under the curve (AUC) compared with other tests for metanephrine, urinary free normetanephrine alone yielded a higher AUC (Table 2). The optimal cutoff for urinary free metanephrine and urinary free normetanephrine had $97.2 \%$ sensitivity and 98.1\% specificity (Supplemental Table S1).

For cases in which urine was not collected properly, each value was compared with corrected urine creatinine value. Urinary free metanephrine and urinary fractionated metanephrine before or after adjustment for 24-hour urinary creatinine yielded simi- lar AUCs to those in the well-collected 24-hour urine group. However, the AUC of urinary free metanephrine was better after adjustment than before (ROC area 0.728 vs. $0.828, P=$ 0.020 ) in the poorly collected 24 -hour urine group. Urinary fractionated metanephrine did not show any difference in AUC even when calibrated with 24-hour urinary creatinine in the poorly collected group (data not shown).

\section{DISCUSSION}

Although analysis of the combined free forms of metanephrine and normetanephrine in urine was not superior to other methods such as those for plasma and urinary fractionated metanephrines, the AUC for urinary free normetanephrine was better than that for urinary fractionated normetanephrine. The lack of statistical difference with combined metanephrine and normetanephrine might be due to the small number of subjects compared with a previous study [9].

Measurement of free metanephrines in urine has advantages

\begin{tabular}{|c|c|c|c|c|c|c|}
\hline \multirow[b]{2}{*}{ Test } & \multirow[b]{2}{*}{ AUC } & \multirow[b]{2}{*}{$95 \% \mathrm{CI}$} & \multicolumn{4}{|c|}{$P$ value } \\
\hline & & & $\begin{array}{l}\text { vs. urinary free } \\
\mathrm{MNs}^{\mathrm{a}}\end{array}$ & $\begin{array}{c}\text { vs. urinary } \\
\text { fractionated } \mathrm{MNs}^{\mathrm{a}}\end{array}$ & $\begin{array}{c}\text { vs. plasma free } \\
\mathrm{MNs}^{\mathrm{a}}\end{array}$ & $\begin{array}{l}\text { vs. urinary } \\
\text { VMA }^{\mathrm{a}}\end{array}$ \\
\hline Urinary free $\mathrm{MN}^{\mathrm{b}}$ & 0.852 & $0.789-0.915$ & & 0.488 & 0.677 & 0.006 \\
\hline Urinary fractionated $\mathrm{MN}^{\mathrm{b}}$ & 0.863 & $0.802-0.925$ & 0.488 & & 0.419 & 0.016 \\
\hline Plasma free $\mathrm{MN}^{\mathrm{c}}$ & 0.842 & $0.779-0.904$ & 0.677 & 0.419 & & 0.005 \\
\hline Urinary free $\mathrm{NMN}^{\mathrm{b}}$ & 0.987 & $0.978-0.996$ & & 0.039 & 0.231 & 0.004 \\
\hline Urinary fractionated $\mathrm{NMN}^{\mathrm{b}}$ & 0.968 & $0.945-0.990$ & 0.039 & & 0.903 & 0.139 \\
\hline Plasma free $\mathrm{NMN}^{\mathrm{c}}$ & 0.970 & $0.939-1.000$ & 0.231 & 0.903 & & 0.207 \\
\hline Urinary free $\mathrm{MN}+\mathrm{NMN}$ & 0.978 & $0.956-1.000$ & & 0.202 & 0.214 & 0.029 \\
\hline Urinary fractionated $\mathrm{MN}+\mathrm{NMN}$ & 0.989 & $0.976-1.000$ & 0.202 & & 0.067 & $<0.001$ \\
\hline Plasma free $\mathrm{MN}+\mathrm{NMN}$ & 0.975 & $0.948-1.000$ & 0.214 & 0.067 & & 0.017 \\
\hline Urinary free $\mathrm{MN} / \mathrm{Cr}$ ratio $^{\mathrm{a}}$ & 0.873 & $0.819-0.926$ & & 0.537 & 0.174 & 0.142 \\
\hline Urinary fractionated $\mathrm{MN} / \mathrm{Cr}$ ratio ${ }^{\mathrm{a}}$ & 0.864 & $0.803-0.925$ & 0.537 & & 0.404 & 0.112 \\
\hline Urinary free $\mathrm{NMN} / \mathrm{Cr}$ ratio $^{\mathrm{a}}$ & 0.983 & $0.972-0.994$ & - & 0.007 & 0.366 & 0.000 \\
\hline Urinary fractionated NMN/Cr ratio ${ }^{a}$ & 0.967 & $0.948-0.987$ & 0.007 & & 0.887 & 0.001 \\
\hline Urinary free $\mathrm{MN} / \mathrm{Cr}$ ratio $+\mathrm{NMN} / \mathrm{Cr}$ ratio & 0.986 & $0.966-1.000$ & - & 0.570 & 0.372 & 0.014 \\
\hline Urinary fractionated $\mathrm{MN} / \mathrm{Cr}$ ratio $+\mathrm{NMN} / \mathrm{Cr}$ ratio & 0.974 & $0.966-1.000$ & 0.570 & - & 0.354 & 0.008 \\
\hline
\end{tabular}


over that of fractionated metanephrines based on their different structures and metabolic processes. The free form of metanephrine is transformed into sulfate-conjugated metanephrine by the action of a specific sulfotransferase isoenzyme, monoaminepreferring sulfotransferase (SULT1A3) [6]. Because SULT1A3 is found in high concentration in the gastrointestinal tract, the level of conjugated metanephrines is affected by food in the gastrointestinal tract [6]. Therefore, urinary free metanephrines can be more useful than urinary conjugated metanephrines in conditions without restriction of diet $[12,13]$. In addition, while conjugated metanephrines in urine require an acid hydrolysis step $[6,14]$, the free form of metanephrines in urine does not undergo this process, reducing analytical interference and showing higher specificity $[10,14]$.

In this study, urinary fractionated metanephrines had high sensitivity and specificity, as previously reported $[13,15,16]$, as did urinary free metanephrines. In addition, as with urinary fractionated metanephrines $[17,18]$, urinary free metanephrines show higher diagnostic accuracy due to adjustment for urinary creatinine when urine collection is poor. In this study, when urinary free metanephrine was poorly collected, the AUC increased after correcting the measurement for urinary creatinine.

Measurement for urinary free metanephrines has several strengths compared to that for plasma free metanephrines. The sensitivity and specificity of plasma free metanephrines were high, ranging from $96 \%-100 \%$ and $85 \%-100 \%$, respectively $[3,10,19]$. However, when measured in a sitting position, the specificity of plasma free metanephrines decreases [11,20]. Considering that most adrenal incidentalomas are examined in outpatient clinics, measurement for urinary free metanephrine that is not affected by posture would be more useful.

It also has been reported that plasma free metanephrines are affected by other diseases [7,15]. Plasma free metanephrines have been found to be elevated in hypothyroidism due to increased norepinephrine secretion, while urinary free metanephrines were not affected [7,15]. As plasma free metanephrines can be extracted by extraneuronal uptake in tissues and metabolized by sulfuric conjugation before being excreted into urine, free metanephrines are less affected in urine than in blood [16].

This study has some limitations. First, it did not include healthy volunteers. Second, the control group included patients who were less likely to develop PPGL after more than 2 years of follow-up. Therefore, people who had not undergone surgery could not be definitively ruled out for PPGL. Third, our study enrolled a small number of subjects who were all analyzed at only one center. To confirm the clinical superiority of urinary free metanephrines measurement, further studies with a larger number of subjects are needed. Nevertheless, it is significant that this study was the first to validate a urinary free metanephrine test and estimate a diagnostic cutoff value for an Asian population.

In summary, urinary free normetanephrine had higher diagnostic efficacy than urinary fractionated normetanephrine, although combined urinary free metanephrine and normetanephrine did not show higher diagnostic accuracy compared to plasma free or urinary fractionated metanephrines.

\section{CONFLICTS OF INTEREST}

No potential conflict of interest relevant to this article was reported.

\section{AUTHOR CONTRIBUTIONS}

Conception or design: J.A., S.M.J., J.H.K. Acquisition, analysis, or interpretation of data: J.A., J.Y.P., G.K., S.M.J., K.Y.H., J.H.K. Drafting the work or revising: J.A., J.H.K. Final approval of the manuscript: J.A., S.Y.L., J.H.K.

\section{ORCID}

Jiyeon Ahn https://orcid.org/0000-0002-5957-3394

Jae Hyeon Kim https://orcid.org/0000-0001-5001-963X

\section{REFERENCES}

1. Lenders JW, Eisenhofer G, Mannelli M, Pacak K. Phaeochromocytoma. Lancet 2005;366:665-75.

2. Pacak K, Eisenhofer G, Ahlman H, Bornstein SR, GimenezRoqueplo AP, Grossman AB, et al. Pheochromocytoma: recommendations for clinical practice from the First International Symposium. October 2005. Nat Clin Pract Endocrinol Metab 2007;3:92-102.

3. Raber W, Raffesberg W, Bischof M, Scheuba C, Niederle B, Gasic S, et al. Diagnostic efficacy of unconjugated plasma metanephrines for the detection of pheochromocytoma. Arch Intern Med 2000;160:2957-63.

4. Young WF Jr. Clinical practice: the incidentally discovered adrenal mass. N Engl J Med 2007;356:601-10.

5. Lenders JW, Duh QY, Eisenhofer G, Gimenez-Roqueplo AP, Grebe SK, Murad MH, et al. Pheochromocytoma and paraganglioma: an Endocrine Society Clinical Practice 
Guideline. J Clin Endocrinol Metab 2014;99:1915-42.

6. Eisenhofer G. Free or total metanephrines for diagnosis of pheochromocytoma: what is the difference? Clin Chem 2001; 47:988-9.

7. Zuo M, Zhen Q, Zhang X, Zou W, Yang X, Tian G, et al. High specificity of spot urinary free metanephrines in diagnosis and prognosis of pheochromocytomas and paragangliomas by HPLC with electrochemical detection. Clin Chim Acta 2018;478:82-9.

8. Woo HI, Yang JS, Oh HJ, Cho YY, Kim JH, Park HD, et al. A simple and rapid analytical method based on solid-phase extraction and liquid chromatography-tandem mass spectrometry for the simultaneous determination of free catecholamines and metanephrines in urine and its application to routine clinical analysis. Clin Biochem 2016;49:573-9.

9. Eisenhofer G, Prejbisz A, Peitzsch M, Pamporaki C, Masjkur J, Rogowski-Lehmann N, et al. Biochemical diagnosis of chromaffin cell tumors in patients at high and low risk of disease: plasma versus urinary free or deconjugated o-methylated catecholamine metabolites. Clin Chem 2018;64:164656.

10. Lee SM, Lee MN, Oh HJ, Cho YY, Kim JH, Woo HI, et al. Development and validation of liquid chromatography-tandem mass spectrometry method for quantification of plasma metanephrines for differential diagnosis of adrenal incidentaloma. Ann Lab Med 2015;35:519-22.

11. Kim HJ, Lee J, Cho YY, Lee SY, Kim JH, Jung BC, et al. Diagnostic accuracy of plasma free metanephrines in a seated position compared with 24-hour urinary metanephrines in the investigation of pheochromocytoma. Endocr J 2015; 62:243-50.

12. de Jong WH, Eisenhofer G, Post WJ, Muskiet FA, de Vries EG, Kema IP. Dietary influences on plasma and urinary metanephrines: implications for diagnosis of catecholamine- producing tumors. J Clin Endocrinol Metab 2009;94:2841-9.

13. Corcuff JB, Chardon L, El Hajji Ridah I, Brossaud J. Urinary sampling for 5HIAA and metanephrines determination: revisiting the recommendations. Endocr Connect 2017;6: R87-98.

14. Peitzsch M, Pelzel D, Glockner S, Prejbisz A, Fassnacht M, Beuschlein F, et al. Simultaneous liquid chromatography tandem mass spectrometric determination of urinary free metanephrines and catecholamines, with comparisons of free and deconjugated metabolites. Clin Chim Acta 2013; 418:50-8.

15. Coulombe P, Dussault JH, Walker P. Catecholamine metabolism in thyroid disease. II. Norepinephrine secretion rate in hyperthyroidism and hypothyroidism. J Clin Endocrinol Metab 1977;44:1185-9.

16. Eisenhofer G, Huynh TT, Hiroi M, Pacak K. Understanding catecholamine metabolism as a guide to the biochemical diagnosis of pheochromocytoma. Rev Endocr Metab Disord 2001;2:297-311.

17. Heron E, Chatellier G, Billaud E, Foos E, Plouin PF. The urinary metanephrine-to-creatinine ratio for the diagnosis of pheochromocytoma. Ann Intern Med 1996;125:300-3.

18. Deboch T, McGrowder DA, Budall SA. Indexing of urinary catecholamines and metanephrines by urinary creatinine levels in the diagnosis of phaeochromocytoma. Trend Med Res 2006;1:113-21.

19. Eisenhofer G, Peitzsch M. Laboratory evaluation of pheochromocytoma and paraganglioma. Clin Chem 2014;60: 1486-99.

20. Sawka AM, Jaeschke R, Singh RJ, Young WF Jr. A comparison of biochemical tests for pheochromocytoma: measurement of fractionated plasma metanephrines compared with the combination of 24-hour urinary metanephrines and catecholamines. J Clin Endocrinol Metab 2003;88:553-8. 